\title{
MODELAGEM DE ATERRAMENTOS ELÉTRICOS PARA FENÔMENOS DE ALTA FREQUÊNCIA E COMPARAÇÃO COM RESULTADOS EXPERIMENTAIS
}

\author{
Rafael Silva Alípio* \\ rafaeledppg . cefetmg.br \\ Márcio Matias Afonso* \\ marciomatiasddes.cefetmg.br
}

\author{
Marco Aurélio de Oliveira Schroeder ${ }^{\dagger}$ \\ schroeder@ufsj.edu.br \\ Tarcísio Antônio Santos de Oliveira* \\ tarcisioddes.cefetmg.br
}

\author{
${ }^{*}$ Departamento de Engenharia Elétrica - DEE \\ Centro Federal de Educação Tecnológica de Minas Gerais (CEFET-MG), Av. Amazonas, CEP 30510-000, \\ Belo Horizonte, MG, Brasil \\ $\dagger$ Departamento de Engenharia Elétrica - DEPEL \\ Universidade Federal de São João del-Rei (UFSJ), Praça Frei Orlando, 170, Centro, CEP 36307-352,
} São João del-Rei, MG, Brasil

\section{RESUMO}

Este artigo apresenta os fundamentos físicos e matemáticos de uma metodologia de cálculo de transitórios eletromagnéticos em aterramentos elétricos, solicitados por fenômenos de alta frequência, por exemplo, aqueles associados à incidência de descargas atmosféricas. As equações resultantes são oriundas da aplicação direta das equações básicas do eletromagnetismo no domínio da frequência, cuja solução é obtida mediante aplicação da técnica numérica Método dos Momentos. Os resultados advindos do modelo proposto são comparados com dados de medição e com resultados de outro modelo, considerado referência na literatura técnica especializada. De tais comparações verifica-se a consistência da modelagem proposta.

PALAVRAS-CHAVE: Aterramentos elétricos, transitórios eletromagnéticos, descargas atmosféricas, modelagem eletromagnética, comparação com medições.

\begin{abstract}
Electrical Grounding Modeling for High Frequency Phenomena and Comparison with Experimental Results This paper presents the physical and mathematical basis of a methodology for electromagnetic transients' calculation in grounding systems, subjected to high frequency phenomena, for example, those associated to lightning. The derived equations arise from the direct application of the electromagnetic basic equations in the frequency domain, whose solution is obtained by the application of the Moment Methods numerical technique. The obtained results by this method are compared both with experimental results and with another model, which is assumed as a reference in the specialized literature. Based on such comparisons, the consistency of the proposed model is verified.
\end{abstract}

KEYWORDS: Grounding, electromagnetic transients, lightning, electromagnetic modeling, comparison with measurements.

\footnotetext{
Artigo submetido em 03/03/2010 (Id.: 01111)

Revisado em 23/05/2010, 14/07/2010

Aceito sob recomendação do Editor Associado Prof. Antonio Carlos Zambroni de Souza
} 


\section{INTRODUÇÃO}

O aterramento elétrico é elemento essencial nos sistemas elétricos e apresenta singular importância para o bom desempenho do sistema aterrado e, principalmente, para segurança de seres vivos (Visacro, 2002). Sua função principal é dispersar a corrente de falta para a terra sem causar diferenças de potenciais ou tensões induzidas perigosas para seres vivos ou que possam danificar equipamentos localizados nas proximidades (Mattos, 2004). O comportamento e projeto de aterramentos para solicitações associadas à frequência industrial estão relativamente bem estabelecidos e seguem, em geral, as orientações da norma internacional IEEE Std. 80 (2000). Contudo, o desempenho do aterramento quando solicitado por correntes impulsivas, advindas da incidência de descargas atmosféricas, pode ser distinto e, em muitos casos, comprometer sensivelmente a eficiência da proteção (Grcev, 2009).

No caso do Brasil, devido às características peculiares do solo, que possui valor médio de resistividade elevado, superior a $1.000 \Omega$.m na maior parte do território, segundo Visacro (2002), o projeto e construção de malhas de aterramento adequadas à segurança de seres vivos e que garantam um bom desempenho do sistema, constituem laboriosa tarefa. Somada às tais características peculiares de solo, deve-se salientar a elevada densidade de incidência de descargas atmosféricas (número de descargas por $\mathrm{km}^{2}$ por ano) para terra no país em relação a outras localidades do mundo (Visacro, 2005). Os aspectos citados conferem importância ao tema aterramentos elétricos, particularmente no entendimento de seu comportamento frente a correntes advindas da incidência de descargas atmosféricas. Isso tem motivado várias pesquisas em âmbito nacional, muitas delas encomendadas pelas próprias concessionárias de energia, tendo em conta o grande prejuízo associado ao fenômeno de incidência de descargas. Em termos de trabalhos nacionais, pode-se citar Portela (1985), Visacro (1992), Soares (1996), Portela (1997), Miranda (2003), Rodrigues (2004), Nogueira (2006), e Alípio (2008a). Vale citar que esses trabalhos têm origem ou guardam semelhança com trabalhos pioneiros desenvolvidos pelos professores Portela e Visacro no início da década de 1990, por exemplo, aquele apresentado em evento nacional no ano de 1993 (Visacro e Portela, 1993). Essa metodologia foi também, posteriormente, aplicada para avaliação de elementos aéreos, por exemplo, em Soares (2001) e Schroeder (2001). Ainda, ressalta-se que recentemente essa metodologia foi bastante explorada e combinada com vários outros modelos de elementos lineares e não lineares em Salari (2006), onde também foi aprimorada para a difícil aplicação em regime não linear, sob o efeito da ionização do solo (Salari e Portela, 2008).

Em âmbito internacional, as primeiras investigações teóricas e experimentais do comportamento impulsivo de eletrodos de aterramento foram conduzidas por Bewley (1934). Em seu trabalho, o autor apresenta uma expressão para cálculo da impedância transiente de cabos contrapesos quando solicitados por um degrau de tensão. Na determinação da referida expressão, o eletrodo de aterramento foi modelado como uma linha de transmissão longa e sem perdas. A partir de metodologia similar, Bellaschi e Armington (1943) avaliaram analiticamente as tensões transitórias no ponto de injeção de eletrodos de aterramento, devido a diferentes formas de onda de corrente. No fim da década de 1940, Sunde apresentou um trabalho pioneiro e publicou um dos livros mais citados na área de aterramentos (Sunde, 1949). Neste trabalho, Sunde apresenta uma análise do aterramento a partir dos conceitos fundamentais das equações de Maxwell e analisa não apenas a resistência de diversas configurações de aterramento, mas também aborda detalhadamente os efeitos indutivos característicos do comportamento impulsivo de eletrodos de aterramento (Sunde, 1949).

No fim da década de 1970 e início da década de 1980, com o aumento da capacidade de processamento dos computadores, novos passos foram possibilitados na ciência e engenharia. Esse aumento de capacidade garantiu, especificamente na modelagem de aterramentos elétricos, importantes desenvolvimentos, por duas razões essenciais:

- Os primeiros modelos, citados anteriormente, assumiam algumas simplificações com o objetivo de chegar a equações e relações simplificadas, que possuíssem solução analítica. Todavia, com a possibilidade de aplicação de técnicas numéricas, equações mais complexas poderiam ser resolvidas;

- Configurações de aterramento mais complexas e extensas poderiam ser modeladas e simuladas com maior facilidade devido ao aumento da memória e velocidade dos computadores.

Os vários modelos matemáticos para representação de sistemas de aterramento no estudo de solicitações devido à incidência de descargas atmosféricas, desenvolvidos desde 1980 até os dias atuais, podem ser classificados segundo dois aspectos basicamente:

i) Metodologia de modelagem adotada:

- Metodologia de teoria de campo (Takashima et alli, 1981; Dawalibi, 1986a; Dawalibi, 1986b; Grcev e Dawalibi, 1990; Dawalibi e Selby, 1993; Grcev, 1996; Olsen e Willis, 1996; Portela, 1997; Visacro e Soares, 2005; Alípio, 2008a; Alípio et alli, 2008b; Salari, 2006); 
- Metodologia de linhas de transmissão (Verma e Mukhedkar, 1980; Mazzetti e Veca, 1983; Velazquez e Mukhedkar, 1984; Menter e Grcev, 1993; Liu et alli, 2001; Lorentzou et alli, 2003; Liu et alli, 2005; He et alli, 2005; Zeng et alli, 2008);

- Metodologia de circuitos elétricos (Meliopoulos e Moharam, 1983; Papalexopoulos e Meliopoulos, 1987; Ramamoorty et alli, 1989; Otero et alli, 1999; Geri, 1999).

ii) Domínio em que o transitório associado é solucionado:

- Domínio do tempo (Verma e Mukhedkar, 1980; Meliopoulos e Moharam, 1983; Mazzetti e Veca, 1983; Velazquez e Mukhedkar, 1984; Papalexopoulos e Meliopoulos, 1987; Ramamoorty et alli, 1989; Menter e Grcev, 1993; Geri, 1999; Liu et alli, 2001; Lorentzou et alli, 2003; Liu et alli, 2005; He et alli, 2005; Zeng et alli, 2008);

- Domínio da frequência (Takashima et alli, 1981; Dawalibi, 1986a; Dawalibi, 1986b; Grcev e Dawalibi, 1990; Dawalibi e Selby, 1993; Grcev, 1996; Olsen e Willis, 1996; Portela, 1997; Otero et alli, 1999; Visacro e Soares, 2005; Alípio, 2008a; Alípio et alli, 2008b).

- Domínios da frequência e do tempo: Salari, 2006.

Os modelos baseados na solução direta das equações de campo são, em geral, desenvolvidos no domínio da frequência. Esse domínio é extremamente adequado para o estudo de campos eletromagnéticos e propagação de ondas. Permite, ainda, a inclusão da variação com a frequência dos parâmetros eletromagnéticos do meio em que o aterramento está inserido. Esse último aspecto é particularmente importante na investigação de sistemas de aterramento inseridos em solos típicos, cujos parâmetros eletromagnéticos (condutividade e permissividade elétricas) têm nítida variação com a frequência, principalmente para espectros elevados (Portela, 1999). Deve-se ressaltar, ainda, que, devido ao fato de se basearem na solução direta das equações de Maxwell, esses modelos são aplicáveis a configurações genéricas e considerados os mais precisos.

As metodologias desenvolvidas no domínio da frequência possuem algumas dificuldades de implementação computacional, por exemplo, na modelagem de efeitos não lineares e no processo de transformação frequência-tempo (Salari e Portela, 2007). Além disso, dependendo do estudo, tais métodos podem demandar um elevado tempo computacional. Em função de tais dificuldades, há uma tendência em se utilizar metodologias mais simplificadas e rápidas desenvolvidas no domínio do tempo, por exemplo, aquelas baseadas na teoria de linhas de transmissão. Todavia, modelos baseados na teoria de linhas assumem que a propagação do campo eletromagnético, guiado pelo eletrodo de aterramento, é ditada pelo modo de propagação transverso eletromagnético (TEM). Essa representação não é fisicamente consistente, tendo em vista que o campo elétrico longitudinal não é desprezível em fenômenos de alta frequência. Deve-se destacar, ainda, que as configurações permitidas por essa abordagem são limitadas a condutores horizontais, não sendo adequada para representação de hastes verticais e configurações genéricas. Pode-se dizer, portanto, que o tratamento da propagação da descarga atmosférica por ondas trafegantes em uma linha de transmissão distancia-se razoavelmente do fenômeno de propagação real.

Metodologias ainda mais simplificadas, baseadas na teoria de circuitos a parâmetros concentrados, modelam os componentes do sistema de aterramento por associações simples de elementos RLC. Esse tipo de modelagem pode ser desenvolvida no domínio da frequência ou ainda utilizando programas computacionais que operem no domínio do tempo, como programas da série ElectroMagnetic Transients Program (EMTP) ou similares (Dommel, 1986). Os modelos oriundos dessa abordagem são baseados em aproximações quase-estáticas, em que os efeitos de propagação são desprezados. Tal aproximação limita a aplicabilidade desta metodologia a frequências da ordem de alguns $\mathrm{kHz}$.

Levando-se em consideração as simplificações associadas às metodologias baseadas nas teorias de circuitos e linhas de transmissão e a melhoria crescente do tempo de processamento dos computadores, o presente trabalho propõe uma metodologia de cálculo de transitórios em aterramentos elétricos, baseada na teoria de campo e desenvolvida no domínio da frequência. A aplicação do Método dos Momentos para solução das equações resultantes possibilita a obtenção dos resultados finais. Este trabalho ainda apresenta, como destaque, a comparação dos resultados obtidos pela aplicação da metodologia proposta com resultados experimentais de eletrodos de aterramentos submetidos a correntes impulsivas. De tal comparação, comprovou-se a consistência do modelo desenvolvido.

Este artigo está organizado da seguinte forma: a descrição e solução do modelo de cálculo são apresentadas na Seção 2. Na Seção 3, os resultados obtidos da aplicação da metodologia proposta são comparados com resultados experimentais e com aqueles obtidos por outro modelo. As principais conclusões deste trabalho e potencialidade do modelo são destacadas na Seção 4. 


\section{MODELO MATEMÁTICO}

O modelo eletromagnético proposto é baseado na idéia fundamental de representação de condutores submetidos a correntes impulsivas, como fonte de dois tipos de corrente: i) longitudinal, ao longo do condutor e ii) transversal, que dele dispersa para o meio circundante. Essa idéia foi originalmente explorada por Visacro (1992) e, posteriormente, expandida por Visacro e Soares (2005) para modelagem de condutores genéricos solicitados por descargas atmosféricas, através de elementos condutores cilíndricos. Neste trabalho, ela é aplicada na modelagem de aterramentos elétricos sob condições transitórios.

Da exploração da idéia básica proposta por Visacro e Soares (2005), naturalmente alguns aspectos foram modificados e outros originalmente incluídos neste trabalho, tendo em conta à formulação original proposta pelos referidos autores. Desses novos aspectos, dois merecem destaque (Alípio, 2008a).

Primeiro, neste trabalho as equações resultantes do modelo são solucionadas a partir da aplicação do método dos momentos (Harrington, 1993). A inclusão desse método na solução computacional permite a adição e utilização de diferentes funções base, se necessário, para modelagem da distribuição de corrente nos eletrodos de aterramento (Alípio, 2008a).

Segundo, a inclusão do efeito da interface solo-ar é realizada mediante aplicação do método das imagens modificado, cujos princípios básicos foram propostos por Takashima et alli (1981). Esse método inclui coeficientes complexos para as imagens. Tais coeficientes são função das características eletromagnéticas do ar e do solo e, também, da frequência, o que torna o método particularmente adequado para aplicações em fenômenos de frequências elevadas.

No modelo proposto, o transitório é solucionado no domínio da frequência. Assim, define-se o sinal de corrente, no domínio do tempo, a ser injetado e, a partir da aplicação de uma transformada direta de Fourier, levanta-se seu espectro de frequência. Da análise desse espectro, determina-se o conjunto de frequências de interesse e a resposta do aterramento é computada para cada uma delas, obtendo-se a função de transferência do sistema. A partir desta última, pode-se determinar a resposta do aterramento no domínio do tempo frente à onda de corrente injetada, por meio de uma transformada inversa de Fourier. O procedimento para obtenção da citada função de transferência é descrito a seguir.

\subsection{Procedimento para cada frequência}

Cada eletrodo é considerado fonte de uma densidade de corrente transversal $I_{T} / L$ que dispersa do condutor em direção ao solo e de uma corrente longitudinal $I_{L}$ que circula ao longo do eletrodo, conforme ilustra a Figura 1 (Visacro e Soares, 2005; Alípio, 2008a). Assume-se que ambas as fontes possuem variações harmônicas no tempo. Essas duas fontes de corrente são necessárias e suficientes para que o sistema sob estudo atenda às condições físicas impostas por todas as equações de Maxwell. A fonte de corrente transversal, devido ao fato de possuir natureza divergente, apresenta efeito elétrico, mas não magnético. A cada fonte de corrente transversal está associado um campo elétrico de natureza conservativa. Este campo gera elevação de potencial em relação ao infinito em pontos genéricos no meio em que o eletrodo se encontra inserido, inclusive nos demais eletrodos e nele mesmo (Alípio, 2008a). Por outro lado, a fonte de corrente longitudinal, devido ao fato de possuir natureza solenoidal, apresenta efeito eletromagnético. A cada fonte de corrente longitudinal está associado um campo magnético, cuja variação no tempo gera um campo elétrico de natureza não-conservativa. $\mathrm{O}$ efeito deste campo elétrico solenoidal se traduz na força eletromotriz induzida em outros eletrodos e, inclusive, no próprio eletrodo fonte (Alípio, 2008a). Pode-se afirmar, portanto, que as duas fontes de corrente descritas são particularmente adequadas para a formulação e solução dos campos eletromagnéticos oriundos de sistemas de eletrodos energizados.

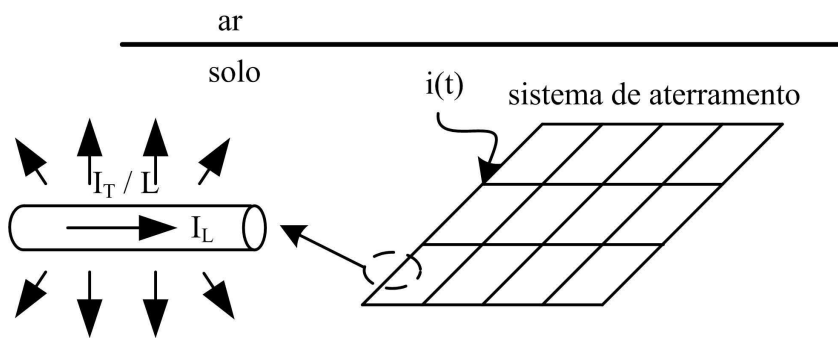

Figura 1: Fontes de corrente em cada eletrodo.

Seja um meio linear, homogêneo e isotrópico, caracterizado por uma constante de propagação $\gamma$, para grandezas com variação harmônica no tempo de velocidade angular $\omega$, sendo

$$
\gamma=\sqrt{j \omega \mu(\sigma+j \omega \varepsilon)}=\alpha+j \beta,
$$

onde $\mu, \sigma$ e $\varepsilon$ são a permeabilidade magnética, a condutividade elétrica e a permissividade elétrica do meio, respectivamente, e $\alpha$ e $\beta$ correspondem à constante de atenuação e à constante de defasamento, respectivamente.

Considere-se inicialmente uma fonte pontual de corrente divergente $I_{T}$ imersa nesse meio. Sendo $r$ a distância entre 
um ponto genérico no meio e a fonte de corrente e $\vec{a}_{r}$ o vetor unitário na direção radial, o potencial escalar elétrico $V$ e o vetor campo elétrico $\vec{E}_{V}$, associados à corrente $I_{T}$, são dados por (Alípio, 2008a):

$$
\left\{\begin{array}{l}
V=\frac{1}{4 \pi(\sigma+j \omega \varepsilon)} \frac{I_{T}}{r} e^{-\gamma r} \\
\vec{E}_{V}=-\vec{\nabla} V=\frac{1}{4 \pi(\sigma+j \omega \varepsilon)} \frac{I_{T}}{r^{2}}(1+\gamma r) e^{-\gamma r} \vec{a}_{r}
\end{array}\right.
$$

Seja agora um eletrodo cilíndrico genérico de comprimento $L_{j}$, designado por eletrodo emissor (índice j) do qual dispersa uma corrente total $I_{T j}$. Assumindo-se que o comprimento $L_{j}$ é suficientemente pequeno, por razões que se tornarão claras adiante no texto, pode-se considerar a corrente total $I_{T j}$ distribuída uniformemente ao longo do eletrodo e o efeito transversal deste pode ser representado como uma soma finita de fontes de corrente transversal pontuais. Dessa maneira, a partir das equações (2), o potencial escalar médio em um ponto $\mathrm{P}$ qualquer, gerado pela corrente transversal total $I_{T j}$ injetada no meio por todo o comprimento do eletrodo, é dado por (Alípio, 2008a):

$$
V=\frac{1}{4 \pi(\sigma+j \omega \varepsilon)} \int_{L_{j}} \frac{I_{T j}}{L_{j}} \frac{e^{-\gamma r}}{r} d l_{j}
$$

Suponha-se agora que o ponto $\mathrm{P}$ está sobre a superfície de outro eletrodo, designado por eletrodo receptor (índice i), de comprimento $L_{i}$, conforme ilustra a Figura 2. Nesta condição, o potencial escalar médio ao longo do eletrodo receptor, devido à densidade de corrente linear que deixa o eletrodo emissor é dado por (Alípio, 2008a):

$$
\left\{\begin{aligned}
V_{i j} & =\frac{1}{L_{i}} \int_{L_{i}} V d l_{i} \\
V_{i j} & =\frac{1}{4 \pi(\sigma+j \omega \varepsilon) L_{j} L_{i}} \int_{L_{i}} \int_{L_{j}} I_{T j} \frac{e^{-\gamma r}}{r} d l_{j} d l_{i}
\end{aligned}\right.
$$

Um procedimento similar pode ser adotado para obtenção dos campos e potenciais associados à fonte de corrente longitudinal. Considere uma corrente longitudinal $I_{L j}$ fluindo ao longo do eletrodo emissor (j). O potencial vetor magnético $\vec{A}$ e o vetor campo elétrico $\vec{E}_{A}$ em um ponto $\mathrm{P}$ qualquer, devido a essa corrente, são dados por (Alípio, 2008a):

$$
\left\{\begin{array}{l}
\vec{A}=\frac{\mu}{4 \pi} \int_{L_{j}} I_{L j} \frac{e^{-\gamma r}}{r} d \vec{l}_{j} \\
\vec{E}_{A}=-j \omega \vec{A}=-j \omega \frac{\mu}{4 \pi} \int_{L_{j}} I_{L j} \frac{e^{-\gamma r}}{r} d \vec{l}_{j}
\end{array}\right.
$$

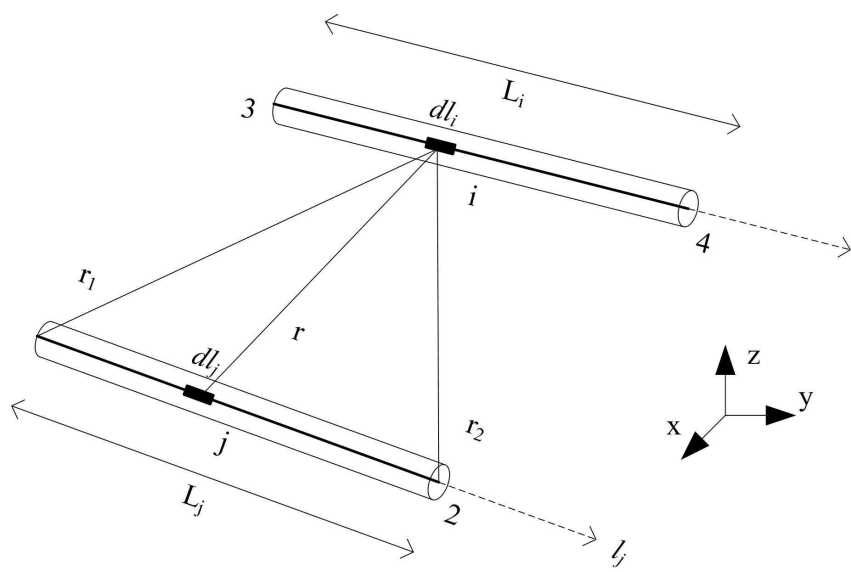

Figura 2: Eletrodo emissor (j) e eletrodo receptor (i).

Suponha-se agora que o ponto $\mathrm{P}$ está sobre a superfície de outro eletrodo, designado por eletrodo receptor (i), de comprimento $L_{i}$, conforme ilustra a Figura 2. Nesta condição, a força eletromotriz induzida no eletrodo receptor, devida à corrente longitudinal que flui que ao longo do eletrodo emissor, é dada por (Alípio, 2008a):

$$
\left\{\begin{aligned}
\Delta V_{i j} & =\int_{L_{i}} \vec{E}_{A} \cdot d \vec{l}_{i} \\
\Delta V_{i j} & =-j \omega \frac{\mu}{4 \pi} \int_{L_{i}} \int_{L_{j}} I_{L j} \frac{e^{-\gamma r}}{r} d \vec{l}_{j} \cdot d \vec{l}_{i}
\end{aligned}\right.
$$

As equações integrais (4) e (6) definem o modelo matemático. A partir da determinação das distribuições de corrente $I_{T j}$ e $I_{L j}$ outras grandezas de interesse podem ser calculadas. Definido o modelo matemático, deve-se proceder à solução do mesmo por meio da aplicação de uma técnica adequada. A escolha dessa técnica é crítica para o desenvolvimento do modelo, posto que ela deve manter um compromisso entre precisão, complexidade do código computacional resultante e tempo de processamento. Antes, porém, julga-se oportuno alguns comentários com relação ao modelo apresentado.

Em condições práticas, para uma ampla faixa de intensidades de corrente, o solo apresenta comportamento linear. Todavia, dependendo da intensidade da corrente imposta ao aterramento, da dimensão dos eletrodos e da resistividade e umidade do solo, o campo elétrico no solo circunvizinho aos eletrodos pode ultrapassar um valor crítico e romper a rigidez dielétrica do meio. Além desse valor crítico o fenômeno de ionização se processa e há a ocorrência de descargas elétricas do eletrodo para o solo. O efeito global da ionização do solo corresponde a uma redução da impedância equivalente de aterramento (Visacro, 2007, Salari e Portela, 2008). Uma vez que o modelo apresentado é baseado na consideração 
de um meio de características lineares, condição necessária para aplicação da transformada de Fourier, há dificuldade de inclusão direta do fenômeno de ionização. Todavia, sua inclusão pode ser realizada de forma indireta, dentro de certa ordem de aproximação, mediante um aumento equivalente no raio do eletrodo de aterramento. Uma metodologia adequada para tal fim pode ser consultada, por exemplo, em Cidrás et alli (2000).

Os valores da condutividade e permissividade elétricas do solo variam com a frequência (Portela, 1999). Assim, para fenômenos transitórios rápidos, nomeadamente aqueles relacionados com a incidência de descargas atmosféricas, pode ser importante a inclusão da variação dos parâmetros eletromagnéticos do solo ao longo do espectro de interesse. Estudos de ordem experimental tentam quantificar, por meio de expressões aproximadas, a dependência desses parâmetros com a frequência (Portela, 1999). No modelo apresentado, uma vez que os cálculos são realizados para cada frequência, a inclusão da variação dos parâmetros pode ser realizada diretamente. Dessa forma, nas expressões de campo obtidas anteriormente, para cada frequência de cálculo determinada, tem-se um valor diferente de condutividade e permissividade elétrica do solo, fornecido por formulação específica, por exemplo, aquela apresentada por Portela (1999).

\subsection{Solução do modelo matemático}

Conforme destacado na subseção anterior, a partir da determinação das distribuições de corrente $I_{T j}$ e $I_{L j}$, outras grandezas de interesse podem ser calculadas. Portanto, o problema consiste em solucionar as equações integrais (4) e (6). Um método adequado para obtenção da solução de tais equações é o Método dos Momentos (Harrington, 1993). A aplicação do Método dos Momentos permite a redução de uma equação integral a um sistema de equações lineares, cuja solução pode ser obtida por meio de algoritmos numéricos de inversão de matrizes (Harrington, 1993).

O procedimento inicial consiste em discretizar o sistema sob estudo, constituído de eletrodos cilíndricos, em $N$ elementos uniformes cada um de comprimento $\ell=\frac{L}{N}$. A razão entre o comprimento $(\ell)$ e o raio do eletrodo é maior que a unidade, de tal forma a permitir a aproximação por correntes filamentares (Harrington, 1993). Neste trabalho, adota-se o comprimento do elemento igual a 10 vezes o raio do eletrodo (Alípio, 2008a). Além disso, o comprimento é suficientemente pequeno de forma que a corrente total que dispersa, assim como a corrente longitudinal, são consideradas uniformes ao longo de um elemento. No entanto, evidentemente, podem variar de um elemento para outro. Essas considerações são fundamentais e importantes no auxílio da escolha de funções base adequadas para representação das distribuições de corrente desconhecidas $I_{T j}(\ell)$ e $I_{L j}(\ell)$. Tal escolha deve ser condizente com a variável física investigada, além de minimizar, sempre que possível, o esforço computacional de implementação.

Desse modo, as distribuições de corrente transversal $I_{T j}(\ell)$ e longitudinal $I_{L j}(\ell)$ ao longo de cada elemento, podem ser representadas por uma combinação linear de $N$ funções base $P_{n}(\ell)$, ou seja, (Alípio, 2008a):

$$
\begin{aligned}
& I_{T j}(\ell)=\sum_{n=1}^{N} I_{T n} P_{n}(\ell) \\
& I_{L j}(\ell)=\sum_{n=1}^{N} I_{L n} P_{n}(\ell)
\end{aligned}
$$

Nas equações (7) e (8) a função base $P_{n}(\ell)$, levando-se em conta considerações anteriores, é definida como:

$$
P_{n}(\ell)=\left\{\begin{array}{l}
0 L<(n-1) \ell \\
1(n-1) \ell \leq L \leq n \ell \\
0 L>n \ell
\end{array}\right.
$$

Nessas mesmas equações, $I_{T n}$ e $I_{L n}$ são coeficientes desconhecidos e correspondem, respectivamente, às correntes transversal e longitudinal do n-ésimo elemento. Para determinação de cada um desses coeficientes são necessárias $\mathrm{N}$ equações linearmente independentes.

Considerando os efeitos transversais, a partir das equações (7) e (9), a segunda das equações (4) pode ser reescrita, para um eletrodo dividido em $\mathrm{N}$ elementos, como (Alípio, 2008a):

$$
V_{i j}=\frac{1}{4 \pi(\sigma+j \omega \varepsilon) \ell_{j} \ell_{i}} \sum_{n=1}^{N} I_{T n} \int_{\ell_{i}} \int_{\ell_{j}} P_{n}(\ell) \frac{e^{-\gamma r}}{r} d l_{j} d l_{i}
$$

A equação (10) fornece o potencial médio no i-ésimo elemento, devido à contribuição dos $\mathrm{N}$ elementos, ou seja:

$$
V_{i}=I_{T 1} z_{T i 1}+I_{T 2} z_{T i 2}+\ldots+I_{T N} z_{T i N}
$$

Mas, levando-se em conta os $N$ elementos que compõem o eletrodo de aterramento, obtêm-se o sistema de equações lineares:

$$
V=Z_{T} I_{T}
$$


onde $V$ corresponde ao vetor elevação de potencial médio em relação ao infinito em cada elemento, $I_{T}$ ao vetor de corrente transversal em cada elemento e $Z_{T}$ é definida como matriz de impedância transversal (Alípio, 2008a). Os termos da matriz de impedância transversal $Z_{T}$ são dados por:

$$
z_{T i j}=\frac{1}{4 \pi(\sigma+j \omega \varepsilon) \ell_{j} \ell_{i}} \int_{\ell_{i}} \int_{\ell_{j}} P_{n}(\ell) \frac{e^{-\gamma r}}{r} d l_{j} d l_{i}
$$

Os termos $z_{T i j}$ correspondem à impedância transversal entre dois elementos, sendo um deles determinado elemento emissor de corrente (j) e outro receptor (i). A impedância $z_{T i j}$ é igual ao potencial médio no elemento receptor, devido à fonte de corrente transversal no elemento emissor, dividido pela corrente transversal deste último. Ela representa a interação eletromagnética entre os diversos elementos e traduz fisicamente os acoplamentos elétricos (capacitivo e condutivo) próprio e mútuo entre eles (Alípio, 2008a).

Raciocínio bastante similar é aplicado para avaliação dos efeitos longitudinais. Portanto, considerando as equações (8) e (9) e aplicando a segunda das equações (6) aos $N$ elementos que compõem o eletrodo de aterramento, obtêm-se o sistema de equações lineares:

$$
\Delta V=Z_{L} I_{L}
$$

onde $\Delta V$ corresponde ao vetor de quedas de tensão em cada elemento, $I_{L}$ ao vetor de corrente longitudinal em cada elemento e $Z_{L}$ é definida como matriz de impedância longitudinal (Alípio, 2008a). Os termos da matriz de impedância longitudinal $Z_{L}$ são dados por:

$$
z_{L i j}=-j \omega \frac{\mu}{4 \pi} \int_{\ell_{i}} \int_{\ell_{j}} P_{n}(\ell) \frac{e^{-\gamma r}}{r} d \vec{l}_{j} \cdot d \vec{l}_{i}
$$

Os termos $z_{L i j}$ correspondem à impedância longitudinal entre dois elementos, sendo um deles determinado elemento emissor de corrente (j) e outro receptor (i). A impedância $z_{L i j}$ é igual à queda de tensão induzida no elemento receptor, devido à fonte de corrente longitudinal no elemento emissor, dividido pela corrente longitudinal deste último. Ela representa a interação eletromagnética entre os diversos elementos e traduz fisicamente os acoplamentos magnéticos (indutivo) próprio e mútuo entre eles (Alípio, 2008a).

Ressalta-se que as impedâncias $z_{T i j}$ e $z_{L i j}$ dependem apenas da geometria do aterramento e das características eletromagnéticas do solo, uma vez que o sistema sob estudo é assumido linear. No cálculo dessas impedâncias, o efeito da interface solo-ar é levado em consideração mediante aplicação do método das imagens modificado (Takashima et alli, 1981).

Assim, as distribuições de corrente transversal e longitudinal, desconhecidas ao longo do eletrodo, são representadas como uma combinação linear de funções base, no caso funções do tipo pulso (Alípio, 2008a). A Figura 3 ilustra a distribuição de corrente ao longo do eletrodo de aterramento, levando-se em consideração as distribuições de correntes propostas.

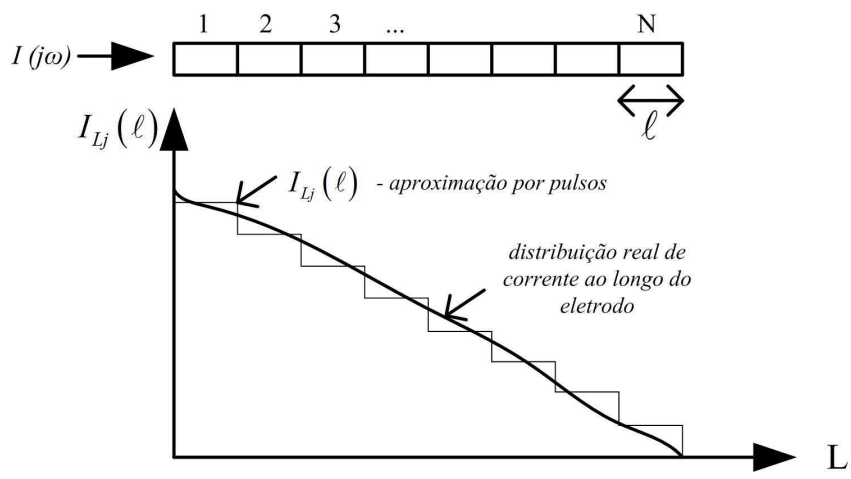

Figura 3: Distribuição de corrente ao longo do eletrodo.

As equações (12) e (14) podem ser reduzidas a uma única equação matricial a partir do estabelecimento de relações nodais entre as grandezas sob estudo. Primeiro, assumem-se o potencial médio em cada elemento igual à média aritmética entre os potenciais nodais e a queda de tensão igual à diferença entre eles. Segundo, a lei de Kirchhoff das correntes é forçada para cada nó do sistema sob estudo, ou seja, a somatória das correntes que entram (ou que deixam) um determinado nó é nula. Da aplicação dessas relações, as equações (12) e (14) podem ser reduzidas a uma única equação matricial da forma $\boldsymbol{A} \boldsymbol{x}=\boldsymbol{b}$ (vide Apêndice A):

$$
\left(\begin{array}{llll}
a_{11} & a_{12} & \ldots & a_{1 p} \\
a_{21} & a_{22} & \ldots & a_{2 p} \\
\vdots & \vdots & \vdots & \vdots \\
a_{p 1} & a_{p 2} & \ldots & a_{p p}
\end{array}\right)\left(\begin{array}{l}
V_{N 1} \\
V_{N 2} \\
\vdots \\
V_{N p}
\end{array}\right)=\left(\begin{array}{l}
1 \\
0 \\
\vdots \\
0
\end{array}\right)
$$

onde:

$\boldsymbol{A}$ é a matriz resultante da aplicação das relações nodais, de dimensão $p x p$, em que $p$ é o número de nós do sistema. Em linhas gerais, ela é resultado de uma combinação das matrizes originais $Z_{T}$ e $Z_{L}$, o que significa que seus termos dependem apenas da geometria do aterramento e das características eletromagnéticas do meio. Sua determinação envolve o cálculo numérico de integrais mediante aplicação do Método de Gauss-Legendre (Campos Filho, 2007). Um 
nó do sistema corresponde à junção entre dois ou mais elementos e, também, àqueles pontos extremos. No caso de um eletrodo horizontal de aterramento, por exemplo, o número de nós é $p=N+1$.

$\boldsymbol{x}$ é o vetor dos potenciais nodais $V_{N}$, de dimensão $p x 1$.

$\boldsymbol{b}$ é o vetor correspondente à injeção de correntes externas, de dimensão $p x 1$. Ele possui o valor 1 nos nós em que há injeção de corrente externa e 0 nos demais nós. No caso da equação (16), considera-se injeção de corrente apenas em um nó.

A partir da solução da equação matricial (16) e obtenção do vetor $\boldsymbol{x}$, as distribuições de corrente transversal e longitudinal são obtidas e, de posse dessas últimas, outras grandezas de interesse na avaliação do comportamento transitório do aterramento podem ser calculadas.

\section{COMPARAÇÃO COM RESULTADOS EXPERIMENTAIS}

Uma ferramenta computacional que implementa a metodologia descrita foi desenvolvida. Os dados de entrada correspondem às coordenadas dos eletrodos e características eletromagnéticas do solo, além de informações sobre a injeção de corrente. A partir desses dados uma série de resultados, nos domínios do tempo e da frequência, são gerados.

Uma etapa fundamental no desenvolvimento de um modelo matemático consiste em sua validação. Nela, a comparação com resultados experimentais apresenta particular importância. Todavia, na literatura técnica especializada e de ampla divulgação, há um número bastante reduzido de trabalhos de cunho experimental na investigação da resposta de aterramentos elétricos a correntes impulsivas. Dentre esses trabalhos, destaca-se aquele conduzido pela Electricité de France (EDF), que realizou algumas medições sistemáticas de eletrodos de aterramento submetidos a correntes de curto tempo de frente, ao longo da década de 1980. Os resultados desse estudo foram apresentados sob a forma de um relatório de pesquisa ao fim dessa mesma década (Rochereau, 1988). No entanto, é importante destacar que apenas configurações mais simples, compostas por eletrodos horizontais e hastes verticais, tipicamente aplicadas em aterramentos de linhas de transmissão e redes de distribuição, foram investigadas.

Tendo em conta esse contexto, é prática comum entre os pesquisadores envolvidos com modelagem de aterramentos elétricos para frequências elevadas, com o objetivo de validar o modelo desenvolvido, adotar uma ou combinação das posturas a seguir:

i) Comparar os resultados obtidos via simulação (pela implementação computacional do modelo proposto) com aqueles de medição, ainda que escassos, disponíveis na literatura. Neste grupo, em praticamente sua totalidade, as medições realizadas pela EDF são tomadas como referência (Rochereau, 1988). Grcev (1996), Otero ett alli (1999), Liu et alli (2005) e Salari (2006) são exemplos de pesquisadores que utilizaram tais medições para aferição de seus modelos de aterramento.

ii) Comparar os resultados obtidos via simulação com aqueles obtidos por outro modelo, tomado como referência. O modelo-referência deve apresentar o mínimo de simplificações possíveis e aplicar rigorosamente as equações básicas do eletromagnetismo (Equações de Maxwell). Neste grupo, é prática geral adotar como referência o modelo desenvolvido por Grcev (1996), referência mundialmente reconhecida em modelagem de aterramentos. Podem-se exemplificar os pesquisadores Geri (1999), Liu et alli (2001) e Salari (2006), que, com fins de validação, compararam resultados obtidos pela metodologia por eles proposta, com aqueles obtidos por Grcev (1996).

Com base nesses comentários inicias, adota-se neste trabalho uma combinação das duas posturas anteriormente descritas para validação do modelo proposto. Duas configurações de eletrodo horizontal são avaliadas.

\section{- Configuração 1}

Consiste em um eletrodo horizontal de $8 \mathrm{~m}$, de cobre (seção $116 \mathrm{~mm}^{2}$ ) e enterrado a $0,6 \mathrm{~m}$ de profundidade no solo. De acordo com Rochereau (1988), estimou-se a

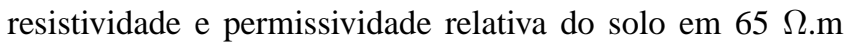
e 15, respectivamente (ver comentários mais à frente no texto). A Figura 4 apresenta a onda de corrente injetada na extremidade do eletrodo, estimada a partir do oscilograma da corrente medida. A Figura 5 ilustra a elevação de potencial medida no ponto de injeção (também estimada dos oscilogramas de medição) e os resultados de simulações obtidos por Grcev (1996) e neste trabalho. De forma geral, o ajuste entre as formas de onda medida e obtida por Grcev foi bastante razoável com aquela calculada neste trabalho. $\mathrm{O}$ erro médio na frente da onda de tensão (de $0 \mu$ s a cerca de $0,2 \mu \mathrm{s}$ ) é da ordem de $4 \%$ e $2 \%$ em relação às curvas medida e obtida por Grcev, respectivamente. Na cauda da onda (de cerca de $0,2 \mu \mathrm{s}$ a $1 \mu \mathrm{s}$ ), o erro médio é da ordem de $7 \%$ e $1 \%$ em relação às curvas medidas e obtida por Grcev, respectivamente. $\mathrm{O}$ valor máximo de tensão medido é $0,4 \%$ superior ao valor calculado neste trabalho.

\section{- Configuração 2}




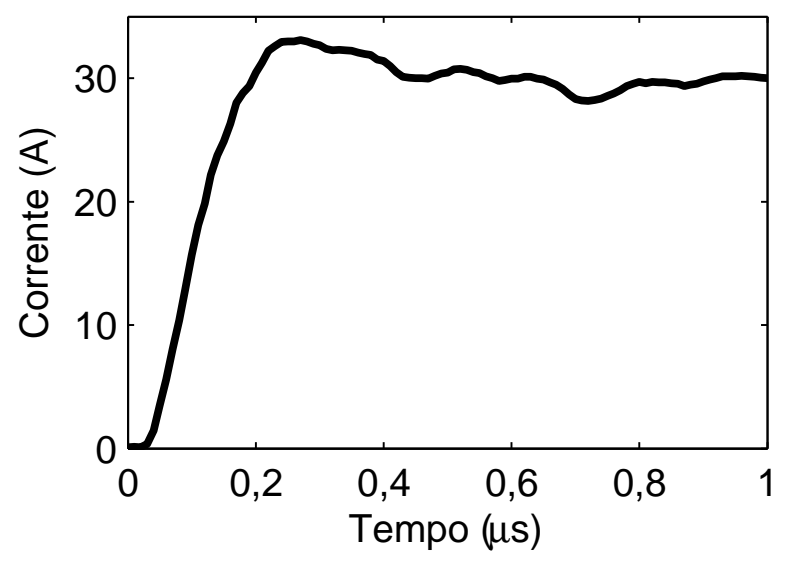

Figura 4: Onda de corrente injetada no eletrodo Configuração 1.

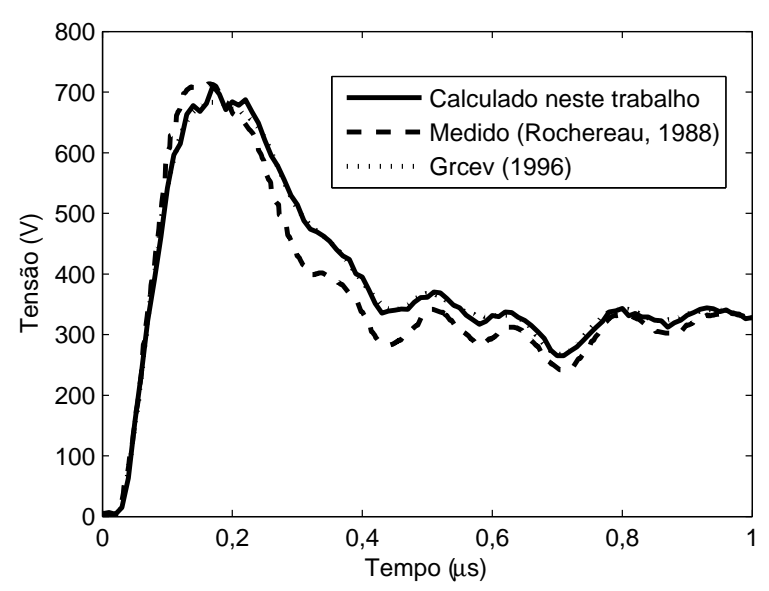

Figura 5: Elevação de potencial no ponto de injeção de corrente - Configuração 1.

Consiste em um eletrodo horizontal de $15 \mathrm{~m}$, de cobre (seção $116 \mathrm{~mm}^{2}$ ) e enterrado a $0,6 \mathrm{~m}$ de profundidade no solo. De acordo com Rochereau (1988), estimou-se a resistividade e permissividade relativa do solo em $70 \Omega . \mathrm{m}$ e 15, respectivamente. A Figura 6 apresenta a onda de corrente injetada na extremidade do eletrodo, estimada dos oscilogramas de medição. A Figura 7 ilustra a elevação de potencial medida no ponto de injeção (estimada dos oscilogramas de medição) e os resultados de simulações obtidos por Grcev (1996) e neste trabalho. Também neste caso observa-se um bom ajuste entre as curvas medida e obtida por Grcev com aquela calculada neste trabalho. O erro médio na frente da onda de tensão (até cerca de 0,2 $\mu$ s, desconsiderando alguns instantes iniciais em que há um desvio razoável entre as curvas calculadas e medida) é da ordem de $4 \%$ em relação à curva experimental. Ainda na frente da onda de tensão, o erro médio em relação aos cálculos de Grcev é da ordem de $1 \%$. Na cauda da onda (de cerca de $0,2 \mu \mathrm{s}$ a $0,7 \mu \mathrm{s}$ ), o erro médio é da ordem de $5 \%$ e $1 \%$ em relação às curvas medida e obtida por Grcev, respectivamente. O valor máximo de tensão medido é $4 \%$ superior ao valor calculado neste trabalho.

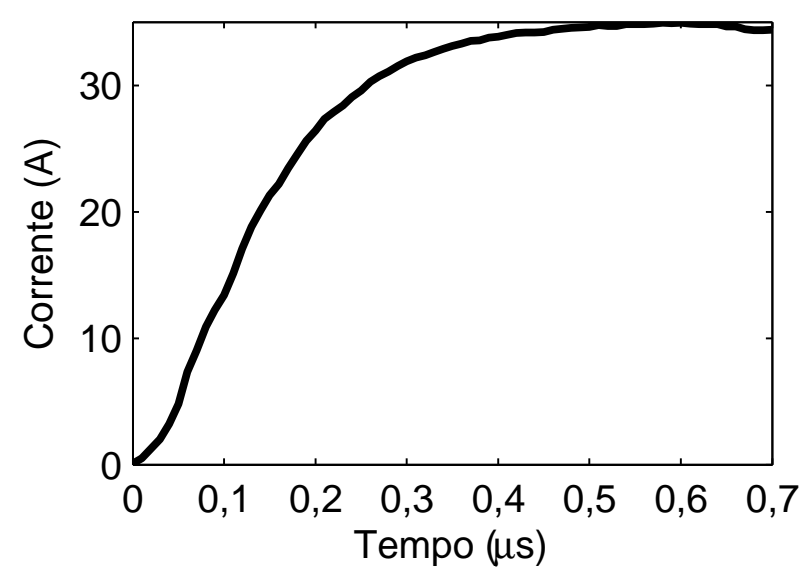

Figura 6: Onda de corrente injetada no eletrodo Configuração 2.

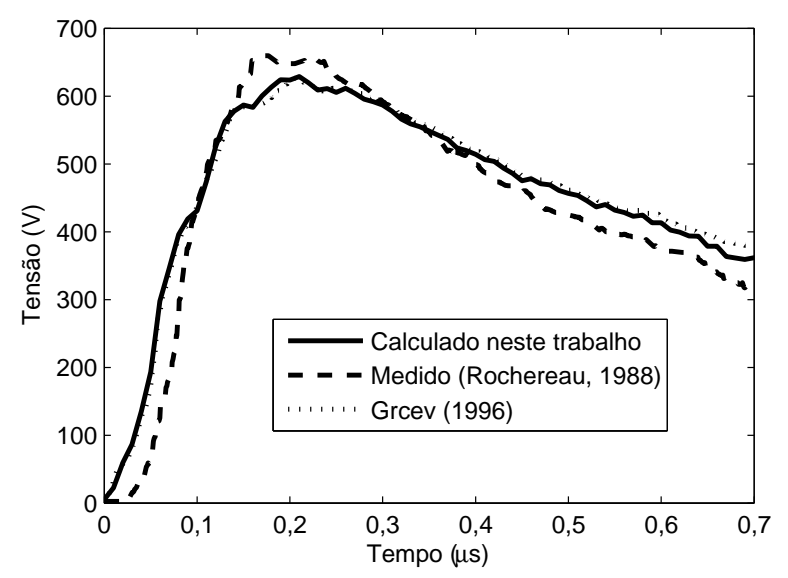

Figura 7: Elevação de potencial no ponto de injeção de corrente - Configuração 2.

Alguns comentários importantes devem ser feitos em relação aos resultados apresentados.

i) A escassez de informações sobre a corrente injetada dificulta a reprodução da forma de onda para fins de simulação. Assim, a onda é estimada diretamente a partir dos oscilogramas de medição (por exemplo, por meio de um código computacional que converta pixels em coordenadas $\mathrm{x}$ e y). Nesse caso, a impossibilidade de reprodução exata da corrente injetada no aterramento pode constituir considerável 
fonte de diferenças entre valores medidos e calculados de determinada grandeza.

ii) Outro aspecto de relevo diz respeito aos parâmetros elétricos do solo (resistividade e permissividade). Segundo relatório da EDF (Rochereau, 1988), durante os estudos experimentais, as características do solo não foram medidas separadamente. A constante dielétrica foi fixada em 15, valor típico de acordo com a umidade do solo. Já a resistividade foi estimada a partir do valor medido de resistência de aterramento. É muito importante ressaltar que, quando uma onda de corrente impulsiva é injetada em um eletrodo de aterramento, a corrente que dele dispersa atinge apenas regiões muito próximas. Tal fato deve-se aos efeitos de propagação, particularmente ao efeito de atenuação, que é significativo no caso de correntes impulsivas, cujas frequências representativas são elevadas. Ainda, tal atenuação é mais acentuada naqueles solos de baixa resistividade (como são os casos aqui analisados -

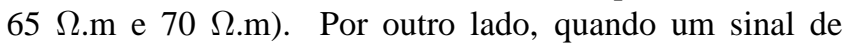
baixa frequência é injetado no aterramento, por exemplo, em uma medição de resistência de aterramento, a corrente associada atinge camadas mais profundas. Nesse caso, os efeitos de atenuação são desprezíveis, mesmo em solos de alta condutividade. Portanto, deduz-se que a "resistividade equivalente" que determinado aterramento "enxerga" pode ser bastante distinta dependendo da frequência representativa da corrente a ele imposta. Assim, em estudos da resposta transitória do aterramento, a estimativa da resistividade do solo a partir da resistência de aterramento pode não ser adequada, principalmente dependendo da estrutura do solo (solo composto por camadas de diferentes resistividades). O mais recomendável neste caso é a caracterização específica do solo. Por exemplo, estimar a estrutura do solo em camadas horizontais pelo método de Frank Wenner. Dessa estrutura, pode-se determinar um valor mais adequado para investigações que envolvam fenômenos de alta frequência. Então, também os parâmetros elétricos adotados na caracterização do solo podem ser fonte de incongruências entre valores medidos e calculados.

iii) Em geral, todo procedimento de medição envolve certas complexidades. No caso de medições em campo, tais complexidades se avolumam. Especificamente em aterramentos, além do circuito de medição (incluído o gerador de impulso e/ou terrômetro, dependendo da aplicação) há outros componentes e fatores importantes que podem influenciar na grandeza medida, tais como: divisores de tensão, eletrodos auxiliares de potencial e corrente e acoplamento eletromagnético entre os condutores (fiação). Deve ficar claro que tais complexidades não são incluídas, em geral, em uma simulação.

Os comentários anteriores permitem fazer algumas inferências ou, caso o termo soe muito forte, algumas suposições.

Primeiro, tendo em conta as incertezas envolvidas no processo de medição, dificuldade de reprodução exata desse processo e ambiguidades referentes aos parâmetros do solo, pode-se afirmar que os erros obtidos entre valores calculados e medidos são suficientemente pequenos, de tal maneira que o modelo é consistente e validado experimentalmente. Obviamente, novos estudos experimentais ainda são importantes e necessários, principalmente para avaliação de configurações mais complexas como, por exemplo, malhas de aterramento.

Segundo, dadas as eventuais diferenças entre a onda de corrente adotada em simulação por Grcev e neste trabalho, as diferenças entre os dois modelos $(\sim 1 \%)$ não é relevante ou significativa. Disso, conclui-se que ambos os modelos geram resultados praticamente idênticos.

Ora, dessa segunda constatação pode surgir o seguinte questionamento: se ambos os modelos geram resultados praticamente idênticos, qual é a vantagem ou real contribuição da modelagem proposta pelos autores? Ambas as metodologias quantificam os acoplamentos eletromagnéticos entre os elementos do aterramento de forma rigorosa por meio da aplicação direta das equações básicas do eletromagnetismo. Também, nos dois casos as equações resultantes são solucionadas a partir da aplicação do Método dos Momentos. Por outro lado, o modelo de Grcev é descrito, em termos de duas grandezas principais: corrente longitudinal ao longo dos condutores e campo elétrico resultante. Assim, a obtenção de grandezas de interpretação e interesse prático imediatos é realizada de forma indireta, por exemplo: i) elevação de potencial no ponto de injeção: obtida da integral de linha do campo elétrico do ponto de injeção até o "terra remoto"; ii) corrente que dispersa dos eletrodos de aterramento: obtida da determinação do campo elétrico normal ao condutor e posterior multiplicação pela condutividade do meio circundante; iii) impedância de aterramento: obtida da razão entre a elevação de potencial no ponto de injeção e a corrente injetada. O modelo proposto neste trabalho é descrito em termos das seguintes grandezas principais: potenciais nodais e correntes transversal e longitudinal de cada elemento. Tais grandezas, que são de interpretação direta, permitem a determinação imediata de grandezas de interesse no projeto de aterramento, por exemplo: i) elevação de potencial no ponto de injeção: corresponde ao potencial do nó de injeção de corrente; ii) corrente que dispersa dos eletrodos de aterramento: corresponde ao vetor de corrente transversal; iii) impedância de aterramento: corresponde à razão entre o potencial do nó de injeção e a corrente injetada. Dessa maneira, entende-se que, principalmente do ponto de vista 
de engenharia, o modelo proposto pode ser uma boa opção em relação ao já consagrado modelo desenvolvido por Grcev (1996).

\section{CONCLUSÕES}

Foram apresentados neste trabalho os detalhes de uma metodologia precisa de cálculo de transitórios em aterramentos elétricos, baseada nas equações básicas do eletromagnetismo, adequada para análise de fenômenos rápidos e lentos. A metodologia resulta da combinação de técnicas numéricas de integração, Método dos Momentos e algoritmos de transformada inversa de Fourier. Uma ferramenta computacional que implementa o método descrito foi desenvolvida. Os resultados obtidos pela aplicação dessa ferramenta foram comparados com resultados de medição e com aqueles obtidos a partir de outro modelo, considerado referência na literatura. De tais comparações constatou-se a validade do modelo desenvolvido.

O modelo descrito neste trabalho apresenta os seguintes destaques:

1) Inclui os acoplamentos eletromagnéticos entre os diversos elementos do aterramento, aspecto de fundamental importância na análise de fenômenos transitórios de alta frequência;

2) Considera os efeitos de propagação, que são significativos no caso de ocorrências rápidas;

3) Permite a inclusão direta da variação dos parâmetros elétricos do solo (condutividade e permissividade elétricas) com a frequência;

4) Permite a inclusão indireta dos efeitos associados à ionização do solo;

5) Fornece grandezas de interpretação e interesse imediatos, tais como elevação de potencial no ponto de injeção e distribuição da corrente de dispersão ao longo da malha;

6) Aplica o Método dos Momentos na solução do modelo matemático apresentado, com a possibilidade de escolha de diferentes funções base para representação das distribuições de corrente ao longo dos eletrodos, o que conduz a um aumento da generalidade de aplicação da modelagem e permite, em alguns casos, a redução do número de elementos resultantes da discretização da malha de aterramento;

7) Apresenta resultados coerentes com aqueles advindos de medições e com aqueles obtidos a partir da aplicação de outra modelagem, considerada referência na literatura.
Julga-se que a ferramenta desenvolvida pode ser um instrumento de valor no auxílio ao estudo, análise e projeto de aterramentos, sobretudo quando é avaliado o desempenho e comportamento dos mesmos frente a ocorrências rápidas. Pode, ainda, servir de subsídio a estudos relacionados ao tema de compatibilidade eletromagnética no cálculo de campos eletromagnéticos e tensões induzidas nas proximidades do aterramento. A ilustração das possibilidades associadas a sua aplicação, na realização de análises de sensibilidade, relacionando diversas variáveis presentes na análise de aterramentos e o seu desempenho nos domínio do tempo e da frequência é objeto de uma publicação futura dos autores.

\section{APÊNDICE A}

O acoplamento das equações (12) e (14) em uma única, (16), que expresse tanto os efeitos transversais quanto longitudinais, é realizado por meio de alguns passos, descritos sucintamente a seguir.

$\mathrm{O}$ primeiro passo corresponde à inversão das matrizes $Z_{T}$ e $Z_{L}$ e obtenção das matrizes admitância transversal $Y_{T}=$ $Z_{T}^{-1}$ e admitância longitudinal $Y_{L}=Z_{L}^{-1}$. A partir dessas relações as equações (12) e (14) podem ser reescritas como (Alípio, 2008a):

$$
\begin{gathered}
I_{T}=Y_{T} V, \\
I_{L}=Y_{L} \Delta V
\end{gathered}
$$

O segundo passo corresponde a dois tipos de relacionamentos (Alípio, 2008a):

O primeiro corresponde à relação entre o potencial médio de cada elemento, dado pelos termos do vetor $V$, e os potenciais nodais do elemento correspondente. Esta relação determina que o potencial médio do elemento é expresso pela média aritmética dos potenciais nodais.

$\mathrm{O}$ segundo corresponde à relação entre a queda de tensão em cada elemento, dada pelos termos do vetor $\Delta V$, e os potenciais nodais do elemento correspondente. Esta relação determina que a queda de tensão no elemento é expressa pela diferença entre os potenciais nodais.

A Figura A ilustra essas duas relações.

A aplicação das duas relações descritas para cada elemento permite reescrever as equações (A1) e (A2) da seguinte forma (Alípio, 2008a):

$$
I_{T}=Y_{T M} V_{N}
$$




$$
I_{L}=Y_{L M} V_{N}
$$

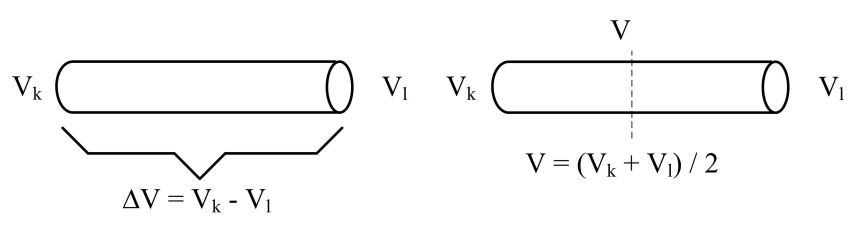

Figura A: Relação entre queda de tensão $(\Delta V)$ e elevação de potencial médio $(V)$ em cada elemento com os potenciais nodais do elemento em questão $\left(V_{k}\right.$ e $\left.V_{l}\right)$.

onde $V_{N}$ corresponde ao vetor de tensões nodais, ou seja, cada elemento do mesmo expressa a tensão em cada nó de conexão entre dois ou mais elementos. Esse vetor tem dimensão $p x 1$, onde $p$ corresponde ao número total de nós. Observa-se que com a transformação das equações (A1) e (A2) para as equações (A3) e (A4), as matrizes $Y_{T}$ e $Y_{L}$ foram substituídas, respectivamente, pelas matrizes $Y_{T M}$ e $Y_{L M}$ (denominadas matrizes modificadas), ambas de dimensão $N x p$. Tal substituição é fruto da transformação dos vetores $V$ e $\Delta V$ em um único vetor de tensões nodais, $V_{N}$.

O terceiro passo corresponde ao estabelecimento de relações entre as correntes transversal e longitudinal (Alípio, 2008a). A lei de Kirchhoff das correntes é avaliada para cada nó do sistema sob estudo, ou seja, a somatória das correntes que entram (ou que deixam) um determinado nó é nula. $\mathrm{Na}$ aplicação de tais relações, a corrente transversal de cada elemento é dividida igualmente entre os nós extremos do elemento em questão, conforme ilustra a Figura A. Por meio desse procedimento, são estabelecidas relações entre as fontes de corrente transversal e longitudinal ao longo de todo o eletrodo. Tais relações permitem reduzir as equações (A3) e (A4) a uma única equação matricial da forma $\boldsymbol{A x}=\boldsymbol{b}$.

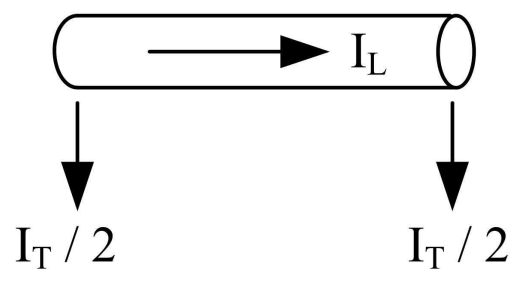

Figura B: Concentração da corrente transversal nos nós do elemento.

\section{REFERÊNCIA BIBLIOGRÁFICA}

Alípio, R. S. (2008a). Modelagem Eletromagnética de Aterramentos Elétricos nos Domínios do Tempo e da Frequência. Dissertação de Mestrado, Programa de Pós-Graduação em Modelagem Matemática e Computacional do Centro Federal de Educação Tecnológica de Minas Gerais, PPGMMC/CEFET-MG, Belo Horizonte - MG.

Alípio, R. S., Schroeder, M. A. O., Afonso, M M. and Oliveira, T. A. S. (2008b). Electromagnetic Fields of Buried Conductors. Proc. of International Conference on Grounding and Earthing (GROUND 2008), Florianópolis, Brazil, pp. 399-402.

Bellaschi, P. L., Armington, R. E. (1943). Impulse and 60-cycle Characteristics of Driven Grounds, Part III. AIEE Transactions, Vol. 62, pp. 334-345.

Bewley, L. V. (1934). Theory and Tests of the Counterpoise. Electrical Engineering, Vol. 53, pp. 1163-1172.

Campos Filho, F. F. (2007). Algoritmos Numéricos. LTC, $2^{a}$ ed., Rio de Janeiro.

Cidrás, J., Otero, A. F. and Garrido, C. (2000). Nodal frequency analysis of grounding systems considering the soil ionization effect. IEEE Transactions on Power Delivery, Vol. 15, ${ }^{\circ} 1$, pp. 103-107.

Dawalibi, F and Selby, A. (1993). Electromagnetic Fields of Energized Conductors. IEEE Transactions on Power Delivery, Vol. 8, $\mathrm{n}^{\circ} 3$, pp. 1275-1284.

Dawalibi, F. (1986a). Electromagnetic Fields Generated by Overhead and Buried Short Conductors, Part I - Single Conductor. IEEE Transactions on Power Delivery, Vol. $1, \mathrm{n}^{\circ} 4$, pp. 105-111.

Dawalibi, F. (1986b). Electromagnetic Fields Generated by Overhead and Buried Short Conductors, Part II - Ground Networks. IEEE Transactions on Power Delivery, Vol. 1, n ${ }^{\circ}$, pp. 112-119.

Dommel, H. W. (1986), Electromagnetic Transients Program (EMTP theory book). Bonneville Power Administration, Vancouver.

Geri, A. (1999). Behavior of Grounding Systems Excited by High Impulse Currents: The Model and its Validation. IEEE Transactions on Power Delivery, Vol. 8, nº3, pp. 1008-1017.

Grcev, L. (1996). Computer Analysis of Transients Voltages in Large Grounding Systems. IEEE Transactions on Power Delivery, Vol. 11, $\mathrm{n}^{\circ} 2$, pp. 815-823. 
Grcev, L. (2009). Impulse Efficiency of Ground Electrodes. IEEE Transactions on Power Delivery, Vol. 24, $\mathrm{n}^{\circ} 1$, pp. 441-451.

Grcev, L. and Dawalibi, F. (1990). An Electromagnetic Model for Transients in Grounding Systems. IEEE Transactions on Power Delivery, Vol. 5, $\mathrm{n}^{\circ} 4$, pp. 1773-1781.

Harrington, R. F. (1993). Field Computation by Moment Methods. IEEE Press, New York.

He, J., Gao, Y., Zeng, R., Zou, J., Liang, X., Zhang, B., Lee, J. and Chang, S. (2005). Effective Length of Counterpoise Wire Under Lightning Current. IEEE Transactions on Power Delivery, Vol. 20, $\mathrm{n}^{\circ} 2$, pp. 1585-1591.

IEEE Guide for Safety in AC Substation Grounding (2000). IEEE Std. 80-2000, 2000.

Liu, Y., Zitnik, M. and Thottappillil, R. (2001). An Improved Transmission-line Model of Grounding System. IEEE Transactions on Electromagnetic Compatibility, Vol. 43, ${ }^{\circ} 3$, pp. 348-355.

Liu, Y., Theethayi, N. and Thottappillil, R. (2005). An Engineering Model for Transient Analysis of Grounding System Under Lightning Strikes: Nonuniform Transmission-line Approach. IEEE Transactions on Power Delivery, Vol. 20, $\mathrm{n}^{\circ} 2$, pp. 722-730.

Lorentzou, M. I., Hatziargyriou, N. D. and Papadias, B. C. (2003). Time Domain Analysis of Grounding Electrodes Impulse Response. IEEE Transactions on Power Delivery, Vol. 18, nº 2, pp. 517-524.

Mattos, M. A. (2004). Técnicas de Aterramento. Okime, Campinas.

Mazzetti, C. and Veca, M. G. (1983). Impulse Behavior of Ground Electrodes. IEEE Transactions on Power Apparatus and Systems, Vol. PAS-102, $\mathrm{n}^{\circ} 9$, pp. 3148-3156.

Meliopoulos, A. P. and Moharam, M. G. (1983). Transient Analysis of Grounding Systems. IEEE Transactions on Power Apparatus and Systems, Vol. PAS-102, ${ }^{\circ} 2$, pp. 389-399.

Menter, F. E. and Grcev, L. (1993). EMTP-based Model for Grounding System Analysis. IEEE Transactions on Power Delivery, Vol. 8, $\mathrm{n}^{\circ} 3$, pp. 1838-1849.

Miranda, J. A. (2003). Simulação de Fenômenos Transitórios em Sistemas de Aterramento. Dissertação de Mestrado, Coordenação dos Programas de
Pós-Graduação da Universidade Federal do Rio de Janeiro, COPPE/UFRJ, Rio de Janeiro - RJ.

Nogueira, R. L. S. (2006). Análise de Sistema de Aterramento sob Solicitações Impulsivas: Otimização e Critérios de Segurança em Aterramentos de Estruturas de Linhas de Transmissão. Dissertação de Mestrado, Coordenação dos Programas de Pós-Graduação da Universidade Federal do Rio de Janeiro, COPPER/UFRJ, Rio de Janeiro - RJ.

Olsen, R. G. and Willis, M. C. (1996). A Comparison of Exact and Quasi-static Methods for Evaluating Grounding Systems at High Frequencies. IEEE Transactions on Power Delivery, Vol. 11, $\mathrm{n}^{\circ} 2$, pp. 1071-1081.

Otero, A. F., Cidrás, J. and del Alamo, J. L. (1999). Frequency-dependent Grounding System Calculation by Means of Conventional Nodal Analysis Technique. IEEE Transactions on Power Delivery, Vol. 8, $\mathrm{n}^{\circ} 3$, pp. 873-878.

Papalexopoulos, A. D. and Meliopoulos, A. P. (1987). Frequency Dependent Characteristics of Grounding Systems. IEEE Transactions on Power Delivery, Vol. 2, n ${ }^{\circ}$, pp. 1073-1081.

Portela, C. (1985). Cálculo de Parâmetros e Comportamento de Malhas de Terra. Curso de Aterramento em Sistemas de Potência, ABNT, Comitê Brasileiro de Eletricidade (COBEI), Rio de Janeiro, Brasil.

Portela, C. (1997). Frequency and Transient Behavior of Grounding Systems - I. Physical and Methodological Aspects. Proc. of the IEEE International Symposium on Electromagnetic Compatibility, Austin, U.S.A., pp. 379-384.

Portela, C. (1999). Measurement and Modeling of Soil Electromagnetic Behavior. Proc. of the IEEE International Symposium on Electromagnetic Compatibility, Seattle, U.S.A., pp. 1004-1009.

Ramamoorty, M., Babu Narayanan, M. M., Parameswaran, S. and Mukhedkar, D. (1989). Transient Performance of Grounding Grids. IEEE Transactions on Power Delivery, Vol. 4, ${ }^{\circ} 4$, pp. 2053-2059.

Rochereau, H. (1988). Response of Earth Electrodes When Fast Fronted Currents are Flowing Out. Bulletin de la Direction des Etudes et Recherches, Electricité de France, serie B, no 2 , pp. 13-22.

Rodrigues, M. G. (2004). Metodologia Aproximada para Simulação do Comportamento Transitório de Malhas 
de Terra e Avaliação da Distribuição de Descargas Atmosféricas. Tese de Doutorado, Coordenação dos Programas de Pós-Graduação da Universidade Federal do Rio de Janeiro, COPPER/UFRJ, Rio de Janeiro - RJ.

Salari, J. C. (2006). Efeito das Descargas Atmosféricas no Desempenho de Linhas de Transmissão - Modelagens nos Domínios do Tempo e da Freqüência. Tese de Doutorado. Coordenação dos Programas de Pós-Graduação de Engenharia da Universidade Federal do Rio de Janeiro, COPPE/UFRJ, Rio de Janeiro - RJ.

Salari, J. C. and Portela, C. (2007). A Methodology for Electromagnetic Transients Calculation An Application for the Calculation of Lightning Propagation in Transmission Lines. IEEE Transactions on Power Delivery, Vol. 22, $\mathrm{n}^{o} 1$, pp. 527-536.

Salari, J. C. and Portela, C. (2008). Grounding systems modeling including soil ionization. IEEE Transactions on Power Delivery, Vol. 23, $\mathrm{n}^{\circ}$ 4, pp. 1939-1945.

Schroeder, M. A. O. (2001). Modelo Eletromagnético para Descontaminação de Ondas de Corrente de Descargas Atmosféricas: Aplicação às Medições da Estação do Moro do Cachimbo. Tese de Doutorado. Centro de Pesquisa e Desenvolvimento em Engenharia Elétrica da Universidade Federal de Minas Gerais, CPDEE/UFMG, Belo Horizonte - MG.

Soares, A. (1996). Modelagem de Linhas de Transmissão para Avaliação de Desempenho frente a Descargas Atmosféricas. Dissertação de Mestrado. Centro de Pesquisa e Desenvolvimento em Engenharia Elétrica da Universidade Federal de Minas Gerais, CPDEE/UFMG, Belo Horizonte - MG.

Soares, A. (2001). Investigação do Comportamento dos Aterramentos típicos de Linhas de Transmissão frente a Descargas Atmosféricas. Tese de Doutorado. Centro de Pesquisa e Desenvolvimento em Engenharia Elétrica da Universidade Federal de Minas Gerais, CPDEE/UFMG, Belo Horizonte - MG.

Sunde, E. D. (1949). Earth Conduction Effects in Transmission Systems. Dover Publications, Inc., New York.

Takashima, T., Nakae, T. and Ishibashi, R. (1981). High Frequency Characteristics of Impedance to Ground and Field Distributions of Ground Electrodes. IEEE Transactions on Power Apparatus and Systems, Vol. PAS-100, n ${ }^{\circ}$, pp. 1893-1900.

Velazquez, R. and Mukhedkar, D. (1984). Analytical Modeling of Grounding Electrodes Transient Behavior. IEEE Transactions on Power Apparatus and Systems, Vol. PAS-103, nº, pp. 1314-1322.
Verma, R. and Mukhedkar, D. (1980). Impulse Impedance of Buried Ground Wire. IEEE Transactions on Power Apparatus and Systems, Vol. PAS-99, $\mathrm{n}^{\circ} 5$, pp. 2003-2007.

Visacro, S. e Portela, C. (1993). Modelagem de Aterramentos Elétricos para Fenômenos Rápidos. Anais do XII Simpósio Nacional de Produção e Transmissão de Energia Elétrica (XII-SNPTEE), Recife, Brasil.

Visacro, S. (1992). Modelagem de Aterramentos Elétricos. Tese de Doutorado, Coordenação dos Programas de Pós-Graduação da Universidade Federal do Rio de Janeiro, COPPER/UFRJ, Rio de Janeiro - RJ.

Visacro, S. (2002). Aterramentos Elétricos. Artiliber, São Paulo.

Visacro, S. (2005). Descargas Atmosféricas: Uma Abordagem de Engenharia. Artiliber, São Paulo.

Visacro, S. (2007). A Comprehensive Approach to the Grounding Response to Lightning Currents. IEEE Transactions on Power Delivery, Vol. 22, $\mathrm{n}^{\circ} 1$, pp. 381-386.

Visacro, S. and Soares, A. (2005). HEM: A Model for Simulation of Lightning-Related Engineering Problems. IEEE Transactions on Power Delivery, Vol. 20, no 2, pp. 1206-1207.

Zeng, R., Gong, X., He, J., Zhang, B. and Gao, Y. (2008). Lightning Impulse Performances of Grounding Grids for Substation Considering Soil Ionization. IEEE Transactions on Power Delivery, Vol. 22, $\mathrm{n}^{\circ} 2$, pp. 667-67. 